\title{
AMPATH Surgical App: Open Appendectomy
}

Joshua Matthews ${ }^{1}$; Manisha Bhatia ${ }^{2}$; Seno Saruni ${ }^{3}$; JoAnna L. Hunter-Squires ${ }^{2,4}$

${ }^{1}$ Indiana University School of Medicine; ${ }^{2}$ Indiana University School of Medicine, Department of Surgery; ${ }^{3}$ Moi Teaching and Referral Hospital; ${ }^{4}$ Moi University School of Medicine, Department of Surgery and Anesthesia

Background/Objective: Due to a geographic shortage of surgical providers within Kenya, doctors without surgical training are expected to complete emergency surgical procedures. The Academic Model Providing Access to Healthcare (AMPATH) surgical team is developing an education module, delivered via mobile phone app and self-made simulators, dedicated to the skills necessary to complete open appendectomy. We hypothesize that our model and curriculum will provide a low-cost method of effectively simulating the open appendectomy.

Methods: After developing a step-based curriculum, an initial prototype of a model for the appendectomy was constructed. Expert academic surgeons from Indiana University were identified to test the prototype by performing an open appendectomy on the model. Feedback on both the model and the procedure was obtained via recorded video and REDCap.

Results: A total of 8 expert surgeons were consulted on the model, each presented with an updated version of the appendectomy model and procedure based on feedback. Experts provided feedback on the model and each substep of the curriculum. Overall, the curriculum was clear with each substep receiving a median score of at least 82 out of 100 for clarity. While the model received lower scores in utility and "realism, expert feedback was incorporated in an iterative process such that latter models demonstrated net improvement in the realism and utility of several substeps, including "the appendectomy".

Conclusion: A low-cost appendectomy model with corresponding curriculum was developed, and refined with expert feedback, to facilitate this project's transition to its next stage - testing on medical trainees. Additionally, the model and curriculum will enable the development of an $\mathrm{Al}$ algorithm to give the learner real-time feedback as they perform the simulation.

Implications: Ultimately, this study may create a platform that increases access to best possible practice and improves outcomes in settings where surgical education is limited. 